\title{
Approximate Analytical Solution to Time-Fractional Damped Burger and Cahn-Allen Equations
}

\author{
A. Esen, N. M. Yagmurlu* and O. Tasbozan \\ Department of Mathematics, İnönü University, Malatya, 44280, Turkey
}

Received: 26 Jan. 2013, Revised: 27 May. 2013, Accepted: 28 May. 2013

Published online: 1 Sep. 2013

\begin{abstract}
The aim of the present paper is to obtain the approximate analytical solutions of time-fractional damped burger and CahnAllen equations by means of the homotopy analysis method (HAM). In the HAM solution, there exists an auxiliary parameter $\hbar$ which provides a convenient way to adjust and check the convergence region of the solution series. In the model problems, an appropriate choice of the auxiliary parameter has been examined for increasing values of time.
\end{abstract}

Keywords: Homotopy analysis method; Approximate analytical solution; Fractional damped burger equation; Fractional Cahn-Allen equation;

\section{Introduction}

In recent years, it has been increasingly noticed that most of the mathematical modelings of many physical phenomena in diverse fields of physics and engineering generally result in nonlinear ordinary or partial differential equations. It is also found out that these kinds of equations model natural phenomena better than those involving only integer order equations. Thus, it has become very important in applied mathematics to investigate and construct exact and numerical solutions of these equations. For example, the HAM which was first proposed by Liao $[1,2]$ is one of the most powerful tool to search the approximate solutions of nonlinear evolution equations (NLEEs). Moreover, the HAM, unlike perturbation techniques, fortunately is not limited to any small physical parameters in the considered equation. Thanks to this useful property, the HAM can tackle with the foregoing restrictions and limitations of perturbation techniques so that it provides us with a more robust tool for analyzing strongly nonlinear problems [3]. This method has been successfully applied for solving several nonlinear problems arising in science and engineering by many authors [1-16] and the references therein. In the present paper, we will apply the HAM to the time-fractional damped Burger and Cahn-Allen equations.
In the literature, one can come across many definitions of the terms fractional integration and differentiation, such as Riemann-Liouville's definition, Caputo's definition and generalized function approach. For instance, the RiemannLiouville integral operator [17] of order $\alpha$ is defined by

$$
\begin{aligned}
& J^{\alpha} f(x)=\frac{1}{\Gamma(\alpha)} \int_{0}^{x}(x-t)^{\alpha-1} f(t) d t \quad(\alpha>0), \\
& J^{0} f(x)=f(x)
\end{aligned}
$$

and its fractional derivative of order $\alpha(\alpha \geq 0)$ is normally characterized by

$$
D^{\alpha} f(x)=\frac{d^{n}}{d x^{n}} J^{n-\alpha} f(x) \quad(\alpha>0, n-1<\alpha<n),
$$

where $n$ is an integer. The Riemann-Liouville integral operator has had a great importance in the development of the theory of fractional derivatives and integrals. Nevertheless, it also has the same disadvantages for fractional differantial equations with initial and boundary conditions. Because of this fact, here we have adopted Caputo's definition[17], which is a modification of Riemann-Liouville definition:

$D^{\alpha} f(x)=J^{n-\alpha} D^{n} f(x)=\frac{1}{\Gamma(n-\alpha)} \int_{0}^{x}(x-t)^{n-\alpha-1} f^{(n)}(t) d t \quad(\alpha>0)$

\footnotetext{
*Corresponding author e-mail: murat.yagmurlu@inonu.edu.tr
} 
where $n$ is an integer. Caputo's integral operator has the following useful characteristic[17]:

$$
J^{\alpha} D^{\alpha} f(x)=f(x)-\sum_{k=0}^{n-1} f^{(k)}\left(0^{+}\right) \frac{x^{k}}{k !} \quad(n-1<\alpha<n) .
$$

For the Caputo derivative, we have

$$
\begin{gathered}
D^{\alpha} c=0(c \text { is a constant }), \\
D^{\alpha} x^{\beta}=\left\{\begin{array}{cc}
0, & \beta \leq \alpha-1, \\
\frac{\Gamma(\beta+1)}{\Gamma(\beta-\alpha+1)} x^{\beta-\alpha}, & \beta>\alpha-1 .
\end{array}\right.
\end{gathered}
$$

In a similar way to integer-order differentiations, Caputo's fractional differentiation is also a linear operation

$$
D^{\alpha}(\lambda f(x)+\mu g(x))=\lambda D^{\alpha} f(x)+\mu D^{\alpha} g(x),
$$

where $\lambda$ and $\mu$ are constants. Moreover Caputo's fractional differentiation also satisfy the so-called Leibnitz rule:

$$
D^{\alpha}(g(t) f(x))=\sum_{k=0}^{\infty}\left(\begin{array}{l}
\alpha \\
k
\end{array}\right) g^{(k)}(t) D^{\alpha-k} f(x) .
$$

For $n$ to be the smallest integer that exceeds $\alpha$, Caputo space-fractional derivative operator of order $\alpha>0$ is described as

$D_{t}^{\alpha}(x, t)=\frac{\partial^{\alpha} u(x, t)}{\partial t^{\alpha}}=\left\{\begin{array}{cc}0, & \text { for } n-1<\alpha<n, \\ \frac{\Gamma(\beta+1)}{\Gamma(\beta-\alpha+1)} x^{\beta-\alpha}, & \text { for } \quad \alpha=n \in N .\end{array}\right.$

In the solution process of the present paper, the Caputo's definition of fractional differentiation is going to be used.

\section{HAM Solutions of the Time-Fractional Damped Burger Equation}

We consider the time-fractional damped burger equation

$$
D_{t}^{\alpha} u+u u_{x}-u_{x x}+\lambda u=0
$$

with the initial condition

$$
u(x, 0)=\lambda x .
$$

To investigate the series solution of Eq. (4) with the initial condition (5), we select the linear operator

$$
\mathscr{L}[\phi(x, t ; p)]=D_{t}^{\alpha}[\phi(x, t ; p)],
$$

having the property

$$
\mathscr{L}[c]=0
$$

where $c$ is a constant. From Eq. (4), we can now describe a nonlinear operator as follows

$$
\mathscr{N}[\phi(x, t ; p)]=\frac{\partial^{\alpha} \phi(x, t ; p)}{\partial t^{\alpha}}+\phi(x, t ; p) \frac{\partial \phi(x, t ; p)}{\partial x}-\frac{\partial^{2} \phi(x, t ; p)}{\partial x^{2}}+\lambda \phi(x, t ; p)
$$

Therefore, we construct the zero-order deformation equation as

$$
(1-p) \mathscr{L}\left[\phi(x, t ; p)-u_{0}(x, t)\right]=p \hbar \mathscr{N}[\phi(x, t ; p)] .
$$

Clearly, if we set $p=0$ and $p=1$, then we obtain

$$
\phi(x, t ; 0)=u_{0}(x, t)=u(x, 0), \phi(x, t ; 1)=u(x, t)
$$

respectively. Thus, as the embedding parameter $p$ increases from 0 to 1 , the solutions also $\phi(x, t ; p)$ vary from the initial value $u_{0}(x, t)$ to the solution $u(x, t)$. If we expand $\phi(x, t ; p)$ in Taylor series with respect to the embedding parameter $p$, we get

$$
\phi(x, t ; p)=u_{0}(x, t)+\sum_{m=1}^{\infty} u_{m}(x, t) p^{m}
$$

where

$$
u_{m}(x, t)=\left.\frac{1}{m !} \frac{\partial^{m} \phi(x, t ; p)}{\partial p^{m}}\right|_{p=0} .
$$

If we choose the auxiliary linear operator, the initial guess and the auxiliary parameter $\hbar$ properly, then the above series converges at $p=1$, and one can easily have

$$
u(x, t)=u_{0}(x, t)+\sum_{m=1}^{\infty} u_{m}(x, t)
$$

which should be one of the solutions of the initially given nonlinear equation, as proved by Liao [2, 5]. If we differentiate Eq. (6) $m$ times with respect to the embedding parameter $p$, we can obtain the $m$ th-order deformation equation

$$
\mathscr{L}\left[u_{m}(x, t)-\chi_{m} u_{m-1}(x, t)\right]=\hbar R_{m}\left(\vec{u}_{m-1}\right),
$$

where

$R_{m}\left(\vec{u}_{m-1}\right)=\frac{\partial^{\alpha} u_{m-1}}{\partial t^{\alpha}}-\frac{\partial^{2} u_{m-1}}{\partial x^{2}}+\lambda u_{m-1}+\sum_{n=0}^{m-1} u_{n}(x, t) \frac{\partial u_{m-1-n}}{\partial x}$

The solution of the $m$ th-order deformation Eq. (9) for $m \geq$ 1 leads to

$$
u_{m}(x, t)=\chi_{m} u_{m-1}(x, t)+\hbar J_{t}^{\alpha}\left[R_{m}\left(\vec{u}_{m-1}\right)\right] .
$$

Using Eq.(10) together with the initial condition given by (5), we successively obtain

$$
\begin{aligned}
& u_{0}(x, t)=\lambda x \\
& u_{1}(x, t)=\frac{2 \hbar \lambda^{2} t^{\alpha} x}{\Gamma(1+\alpha)} \\
& u_{2}(x, t)=\frac{2 \hbar \lambda^{2} t^{\alpha} x}{\Gamma(1+\alpha)}+\frac{2 \hbar^{2} \lambda^{2} t^{\alpha} x}{\Gamma(1+\alpha)}+\frac{6 \hbar^{2} \lambda^{3} t^{2 \alpha} x}{\Gamma(1+2 \alpha)}
\end{aligned}
$$


etc. As a result, the series solutions expressed by HAM can be written in the following form

$$
u(x, t)=u_{0}(x, t)+u_{1}(x, t)+u_{2}(x, t)+u_{3}(x, t)+\ldots
$$

To demonstrate the efficiency of the present method, we compare the HAM solutions of time-fractional damped burger equation given by Eq. (4) for $\alpha=1$ with the exact solutions [18]

$$
u(x, t)=\frac{\lambda x}{2 \exp (\lambda t)-1}
$$

From now on, for all the computations the value of $\lambda$ will be taken as 1 . Remember that our HAM solution series contains the auxiliary parameter $\hbar$ which provides us with a simple tool to adjust and check the convergence of the solution series. To obtain an appropriate range for $\hbar$, we consider the so-called $\hbar$-curve to select a suitable value of $\hbar$ which ensures the convergence of the solution series, as pointed by Liao [2], by discovering the valid region of $\hbar$ corresponding to the line segments nearly parallel to the horizontal axis. In Fig. 1 , we demonstrate the $\hbar$-curves of $u(0.5,1)$ given by 10 th-order HAM solution (12)and for various values of $\alpha$ parametres. It can be seen from the figure that the valid range of $\hbar$ is approximately $-0.5 \leq$ $\hbar \leq-0.1$.

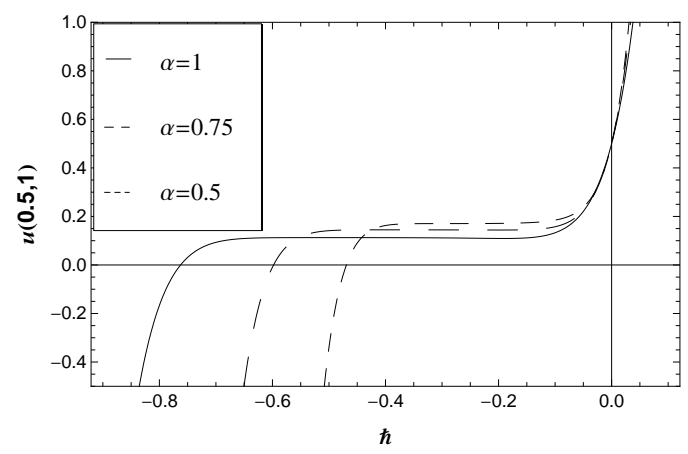

Fig. 1: The $\hbar$-curves of 10th-order approximate solution obtained by the HAM

Fig. 2 shows the numerical solutions of $u(x, t)$ at the point $x=0.5$ for the time interval $0 \leq t \leq 1$ for $\hbar=-1$, $\hbar=-0.75$ and $\hbar=-0.4$ obtained by the HAM for $\alpha=1$ and analytical solutions. Between the times $t=0$ and $t=1$, it can be seen from Fig. 2 that the choice of $\hbar=-0.4$ is a suitable value at $x=0.5$.

In Table 1, the results obtained by the HAM for various values of $\alpha$ by the 10th-order approximate solution of $u(x, t)$ for $\hbar=-0.4$ have been presented.

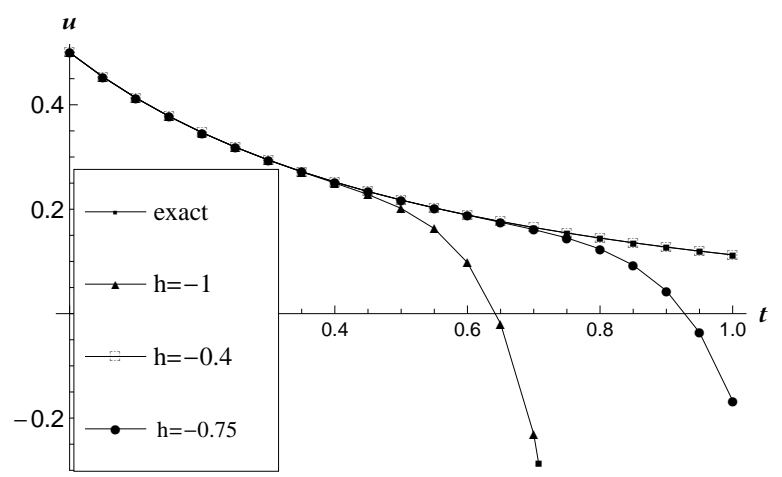

Fig. 2: A comparison of the results obtained by the HAM for various values of $\hbar$ and $\alpha=1$ by 10th-order approximate solution of $u(x, t)$ with the exact solutions at $x=0.5$

Table 1: The results obtained by the HAM for various values of $\alpha$ by 10th-order approximate solution of $u(x, t)$

\begin{tabular}{|c|c|c|c|c|c|}
\hline \multirow[b]{2}{*}{$t$} & \multirow[b]{2}{*}{$x$} & \multirow{2}{*}{$\begin{array}{c}\alpha=0.5 \\
\text { Numerical }\end{array}$} & \multirow{2}{*}{$\begin{array}{c}\alpha=0.75 \\
\text { Numerical }\end{array}$} & \multicolumn{2}{|c|}{$\alpha=1.0$} \\
\hline & & & & Numerical & Exact \\
\hline \multirow[t]{5}{*}{0.25} & 0.2 & 0.099490 & 0.110974 & 0.127574 & 0.127547 \\
\hline & 0.4 & 0.198979 & 0.221948 & 0.255148 & 0.255094 \\
\hline & 0.6 & 0.298468 & 0.332922 & 0.382722 & 0.382641 \\
\hline & 0.8 & 0.397958 & 0.443896 & 0.510296 & 0.510188 \\
\hline & 1.0 & 0.497447 & 0.554870 & 0.637870 & 0.637734 \\
\hline \multirow[t]{5}{*}{0.50} & 0.2 & 0.083409 & 0.083660 & 0.086991 & 0.087053 \\
\hline & 0.4 & 0.166819 & 0.167320 & 0.173982 & 0.174107 \\
\hline & 0.6 & 0.250228 & 0.250979 & 0.260973 & 0.261160 \\
\hline & 0.8 & 0.333637 & 0.334639 & 0.347964 & 0.348213 \\
\hline & 1.0 & 0.417046 & 0.418299 & 0.434954 & 0.435267 \\
\hline \multirow[t]{5}{*}{0.75} & 0.2 & 0.074103 & 0.068179 & 0.061762 & 0.061843 \\
\hline & 0.4 & 0.148207 & 0.136358 & 0.123524 & 0.123686 \\
\hline & 0.6 & 0.222310 & 0.204537 & 0.185287 & 0.185529 \\
\hline & 0.8 & 0.296414 & 0.272716 & 0.247049 & 0.247372 \\
\hline & 1.0 & 0.370517 & 0.340895 & 0.308811 & 0.309215 \\
\hline \multirow[t]{5}{*}{1.00} & 0.2 & 0.065038 & 0.057924 & 0.044999 & 0.045078 \\
\hline & 0.4 & 0.130075 & 0.115849 & 0.089999 & 0.090160 \\
\hline & 0.6 & 0.195112 & 0.173773 & 0.134998 & 0.135240 \\
\hline & 0.8 & 0.260150 & 0.231697 & 0.179997 & 0.180320 \\
\hline & 1.0 & 0.325187 & 0.289621 & 0.224997 & 0.225400 \\
\hline
\end{tabular}
for $\hbar=-0.4$

\section{HAM Solutions of the Time-Fractional Cahn-Allen Equation}

We consider the fractional Cahn-Allen equation

$$
D_{t}^{\alpha} u-u_{x x}+u^{3}-u=0
$$

with the initial condition

$$
u(x, 0)=\frac{1}{1+\exp \left(-\frac{\sqrt{2}}{2} x\right)} .
$$

To investigate the series solution of Eq. (14) with the initial condition (15), we choose the linear operator

$$
\mathscr{L}[\phi(x, t ; p)]=D_{t}^{\alpha}[\phi(x, t ; p)],
$$


having the property

$$
\mathscr{L}[c]=0
$$

where $c$ is constant. From Eq. (14), we can now describe a nonlinear operator as

$\mathscr{N}[\phi(x, t ; p)]=\frac{\partial^{\alpha} \phi(x, t ; p)}{\partial t^{\alpha}}-\frac{\partial^{2} \phi(x, t ; p)}{\partial x^{2}}+\phi(x, t ; p)^{3}-\phi(x, t ; p)$

As a result, we establish the zero-order deformation equation as follows

$$
(1-p) \mathscr{L}\left[\phi(x, t ; p)-u_{0}(x, t)\right]=p \hbar \mathscr{N}[\phi(x, t ; p)] .
$$

Clearly, by setting $p=0$ and $p=1$, we obtain

$$
\phi(x, t ; 0)=u_{0}(x, t)=u(x, 0), \phi(x, t ; 1)=u(x, t)
$$

respectively. Thus, as the embedding parameter $p$ increases from 0 to 1 , the solutions $\phi(x, t ; p)$ vary from the initial value $u_{0}(x, t)$ to the solution $u(x, t)$. Expanding $\phi(x, t ; p)$ in Taylor series with respect to the embedding parameter $p$, we obtain

$$
\phi(x, t ; p)=u_{0}(x, t)+\sum_{m=1}^{\infty} u_{m}(x, t) p^{m},
$$

where

$$
u_{m}(x, t)=\left.\frac{1}{m !} \frac{\partial^{m} \phi(x, t ; p)}{\partial p^{m}}\right|_{p=0} .
$$

If the auxiliary linear operator, the initial guess and the auxiliary parameter $\hbar$ are properly chosen, then the above series converges at $p=1$, and one has

$$
u(x, t)=u_{0}(x, t)+\sum_{m=1}^{\infty} u_{m}(x, t)
$$

which should be one of the solutions of the initially given nonlinear equation, as proved by Liao [2, 5]. Differentiating Eq.(16) $m$ times with respect to the embedding parameter $p$, we obtain the $m$ th-order deformation equation

$$
\mathscr{L}\left[u_{m}(x, t)-\chi_{m} u_{m-1}(x, t)\right]=\hbar R_{m}\left(\vec{u}_{m-1}\right),
$$

where

$R_{m}\left(\vec{u}_{m-1}\right)=\frac{\partial^{\alpha} u_{m-1}}{\partial t^{\alpha}}-\frac{\partial^{2} u_{m-1}}{\partial x^{2}}-u_{m-1}+\sum_{n=0}^{m-1}\left(\sum_{k=0}^{n} u_{k}(x, t) u_{n-k}(x, t)\right) u_{m-1-n}(x, t)$.

The solution of the $m$ th-order deformation Eq. (19) for $m \geq 1$ leads to

$$
u_{m}(x, t)=\chi_{m} u_{m-1}(x, t)+\hbar J_{t}^{\alpha}\left[R_{m}\left(\vec{u}_{m-1}\right)\right] .
$$

Using Eq.(20) with the initial condition given by (14), we successively obtain

$$
\begin{aligned}
& u_{0}(x, t)=\frac{1}{1+\exp \left(-\frac{\sqrt{2}}{2} x\right)} \\
& u_{1}(x, t)=-\frac{3 \hbar t^{\alpha}}{4\left(1+\cosh \left(\frac{x}{\sqrt{2}}\right)\right) \Gamma(1+\alpha)}
\end{aligned}
$$

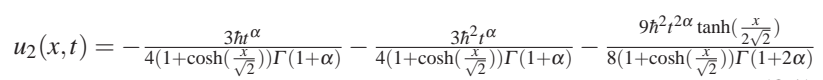

etc. Therefore, the series solutions expressed by HAM can be written in the form

$$
u(x, t)=u_{0}(x, t)+u_{1}(x, t)+u_{2}(x, t)+u_{3}(x, t)+\ldots
$$

To demonstrate the efficiency of the method, we compare the HAM solutions of time-fractional damped burger equation by Eq. (14) for $\alpha=1$ with exact solutions [19]

$$
u(x, t)=\frac{1}{1+\exp \left(-\frac{\sqrt{2}}{2}\left(x+\frac{3 \sqrt{2}}{2} t\right)\right)}
$$

In Fig. 3 , we demonstrate the $\hbar$-curves of $u(0.5,1)$ given by 5 th-order HAM solution (22) for various $\alpha$ parametres. It can be seen from the figure that the valid range of $\hbar$ is approximately $-1.0 \leq \hbar \leq-0.3$.

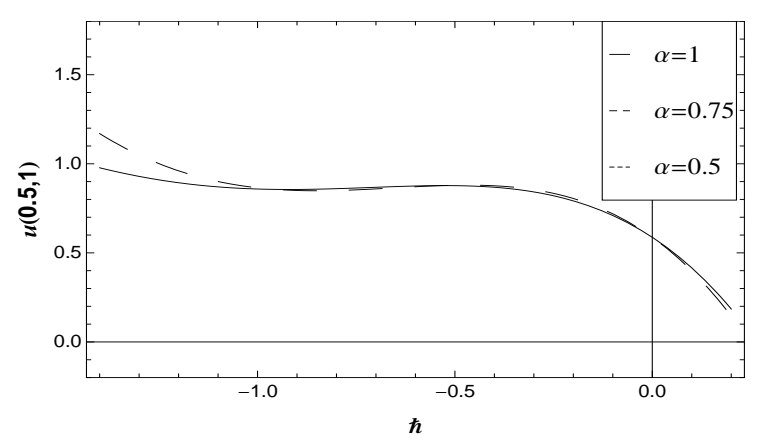

Fig. 3: The $\hbar$-curves of 5 th-order approximate solution obtained by the HAM

Fig. 4 shows the numerical solutions of $u(x, t)$ at $x=0.5$ for the time interval $0 \leq t \leq 1$ for $\hbar=-1.2$, $\hbar=-0.75$ and $\hbar=-0.1$ obtained by the HAM for $\alpha=1$ and analytical solutions. Between the times $t=0$ and $t=1$, it can be seen from Fig. 4 that the choice of $\hbar=-0.75$ is a suitable value at $x=0.5$.

Table 2 tabulates the results obtained by the HAM for various values of $\alpha$ by 5th-order approximate solution of $u(x, t)$ for $\hbar=-0.75$. 


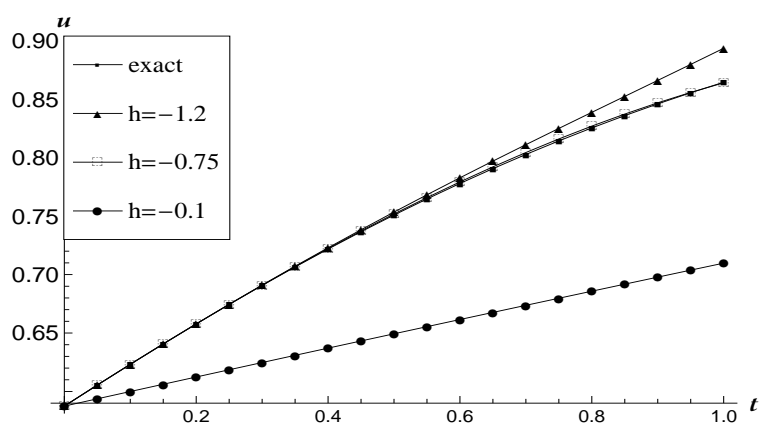

Fig. 4: The results obtained by the HAM for various $\hbar$ by 5 th-order approximate solution of $u(x, t)$, in comparison with the exact solutions at $x=0.5$

Table 2: The results obtained by the HAM for various $\alpha$ by 5 th-order approximate solution of $u(x, t)$ for $\hbar=-0.75$

\begin{tabular}{|c|c|c|c|c|c|}
\hline \multirow{2}{*}{$t$} & \multirow{2}{*}{$x$} & \multirow{2}{*}{$\begin{array}{c}\alpha=0.5 \\
\text { Numerical }\end{array}$} & \multirow{2}{*}{$\begin{array}{c}\alpha=0.75 \\
\text { Numerical }\end{array}$} & \multicolumn{2}{|c|}{$\alpha=1.0$} \\
\hline & & & & Numerical & Exact \\
\hline \multirow[t]{5}{*}{0.25} & 0.2 & 0.720644 & 0.670868 & 0.626257 & 0.626311 \\
\hline & 0.4 & 0.746049 & 0.700739 & 0.658755 & 0.658776 \\
\hline & 0.6 & 0.770000 & 0.728997 & 0.689823 & 0.689817 \\
\hline & 0.8 & 0.792442 & 0.755527 & 0.719263 & 0.719238 \\
\hline & 1.0 & 0.813346 & 0.780257 & 0.746931 & 0.746892 \\
\hline \multirow[t]{5}{*}{0.50} & 0.2 & 0.773627 & 0.747948 & 0.710231 & 0.709183 \\
\hline & 0.4 & 0.794027 & 0.772052 & 0.738428 & 0.737467 \\
\hline & 0.6 & 0.813440 & 0.794543 & 0.764769 & 0.763915 \\
\hline & 0.8 & 0.831778 & 0.815424 & 0.789202 & 0.788463 \\
\hline & 1.0 & 0.848957 & 0.834708 & .811714 & 0.811090 \\
\hline \multirow[t]{5}{*}{0.75} & 0.2 & 0.801911 & 0.799847 & 0.782775 & 0.780130 \\
\hline & 0.4 & 0.819719 & 0.818642 & 0.805400 & 0.803425 \\
\hline & 0.6 & 0.837095 & 0.836326 & 0.826175 & 0.824807 \\
\hline & 0.8 & 0.853776 & 0.852895 & 0.845168 & 0.844314 \\
\hline & 1.0 & 0.869519 & 0.868329 & 0.862458 & 0.862013 \\
\hline \multirow[t]{5}{*}{1.00} & 0.2 & 0.817043 & 0.831645 & 0.839665 & 0.837728 \\
\hline & 0.4 & 0.834311 & 0.846460 & 0.856228 & 0.856048 \\
\hline & 0.6 & 0.851631 & 0.861016 & 0.871456 & 0.872613 \\
\hline & 0.8 & 0.868449 & 0.875147 & 0.885455 & 0.887523 \\
\hline & 1.0 & 0.884298 & 0.888655 & 0.898291 & 0.900886 \\
\hline
\end{tabular}

\section{Conclusion}

In this paper, approximate analytical solutions of time-fractional damped burger and Cahn-Allen equations have been obtained by a successful application of the HAM. It has been also seen that the HAM solution of the problem converges very rapidly to the exact one by selecting an appropriate auxiliary parameter $\hbar$. In conclusion, this study shows that the HAM is a robust and efficient technique in finding the approximate analytical solutions of fractional damped burger equation and also many other nonlinear evolution equations arising in science and engineering. The authors are grateful to the anonymous referees for their careful checking of the details and for their helpful comments that contributed to the improvement of this paper.

\section{References}

[1] S. J. Liao, The proposed homotopy analysis tecnique for the solution of nonlinear problems, Ph.D thesis, Shanghai Jiao Tong University, (1992).

[2] S. J. Liao, Beyond Perturbation: Introduction to the Homotopy Analysis Method, Chapman and Hall/CRC Press, Boca Raton, (2003).

[3] S. J. Liao, Homotopy analysis method: A new analytical technique for nonlinear problems, Commun. Nonlinear Sci. Numer. Simulat, 2, 95-100 (1997).

[4] S. J. Liao, On the homotopy analysis method for nonlinear problems, Appl. Math. Comput., 147, 499513 (2004).

[5] S. J. Liao, Notes on the homotopy analysis method: Some definitions and theorems, Commun. Nonlinear Sci. Numer. Simulat., 14 983-997 (2009).

[6] S. Abbasbandy, The application of homotopy analysis method to solve a generalized Hirota-Satsuma coupled KdV equation, Phys. Lett. A, 361, 478-483 (2007).

[7] E. Babolian, J. Saeidian, Analytic approximate solutions to Burgers, Fisher, Huxley equations and two combined forms of these eqautions, Commun. Nonlinear. Sci. Numer. Simulat., 14, 1984-1992 (2009).

[8] A. Fakhari, G. Domairry, Ebrahimpour, Approximate explicit solutions of nonlinear BBMB equations by homotopy analysis method and comparison with the exact solution, Phys. Lett. A. 368, 64-68 (2007).

[9] M. M. Rashidi, G. Domairry, A. DoostHosseini, S. Dinarvand, Explicit Approximate Solution of the Coupled KdV Equations by using the Homotopy Analysis Method, Int. Journal of Math. Analysis, 2, 581-589 (2008).

[10] M. Inc, On numerical solution of Burgers equation by homotopy analysis method, Phys Lett A, 372, 356360 (2008).

[11] M. Kurulay, A. Secer and M. A. Akinlar, A New Approximate Analytical Solution of KuramotoSivashinsky Equation Using Homotopy Analysis Method, Appl. Math. Inf. Sci., 7, 267-271 (2013).

[12] O. Tasbozan, A. Esen and N. M. Yagmurlu, Approximate analytical solutions of fractional coupled $\mathrm{mKdV}$ equation by homotopy analysis method, Open Journal of Applied Science, 2, 193-197 (2012).

[13] L. Song and H. Q. Zhang, Application of homotopy analysis method to fractional KdV-Burgers-Kuramoto equation, Phys. Lett. A, 367, 88-94 (2007).

[14] S. Abbasbandy, The application of homotopy analysis method to nonlinear equations arising in heat transfer, Phys. Lett. A, 360, 109-113 (2006).

[15] T. Hayat and M. Sajid, On analytic solution for thin film flow of a forth grade fluid down a vertical cylinder, Phys. Lett. A, 361, 316-322 (2007). 
[16] S. J. Liao and A. Y. Tan, A general approach to obtain series solutions of nonlinear differential equations, Commun. Nonlinear. Sci. Numer. Simulat., 14, 983997 (2009).

[17] L. Podlubny, Fractional Differantial Equations, Academic Press, London, (1999).

[18] Y. Peng and W. Chen, A new similarity solution of the Burgers equation with linear damping, Czech. J, Phys. 56, 317-428 (2008).

[19] G. Hariharan, Haar Wavelet Method for Solving Cahn-Allen Equation, Applied Mathematical Sciences, 3, 2523-2533 (2009).

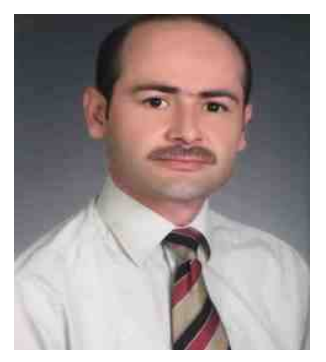

\begin{abstract}
Alaatin ESEN received his diploma in mathematics from the Inonu University in 1994. He has completed his M.Sc. and Ph.D. degrees in applied mathematics. $\mathrm{He}$ is currently studying about the numerical solutions of a wide range of partial differential equations.
\end{abstract}

He has many research papers published in various national and international journals. $\mathrm{He}$ has given talks and conferences. His research interests include finite difference methods, finite element methods, computational methods and algorithms.

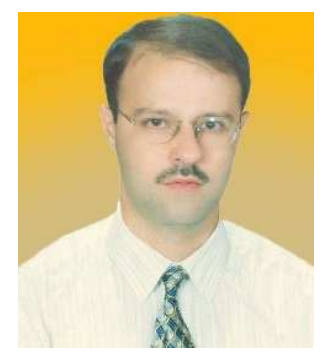

Nuri $\begin{array}{r}\text { Murat } \\ \text { received }\end{array}$
his diploma in computer
engineering from the
Middle East Technical
University. Then he has
completed his Ph.D. degree
in applied mathematics. He is
currently studying about the
approximate solutions of both ordinary and partial two-dimensional differential equations. He contributed national and international talks and conferences. He is primarily interested in numerical methods, computer science and combination of the both.

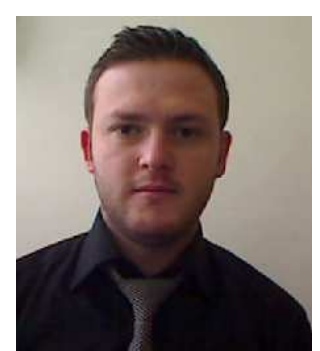

FEM etc.
Orkun TABOZAN graduated from the department of Mathematics of the Afyon Kocatepe University. He has completed his M.Sc. degree in applied mathematics. He is currently studying his Ph.D. thesis. His main interest areas include analytical solutions, fractional differential equations, HAM, 